\title{
A deeper look at the dust attenuation law of star-forming galaxies at high redshift
}

\author{
M. Tress ${ }^{1}$, I. Ferreras ${ }^{1,2,3 \star}$, P. G. Pérez-González ${ }^{4,5}$, A. Bressan ${ }^{6}$, \\ G. Barro ${ }^{7}$, H. Domínguez-Sánchez ${ }^{8}$, C. Eliche-Moral ${ }^{5}$ \\ 1 Mullard Space Science Laboratory, University College London, Holmbury St Mary, Dorking, Surrey RH5 6NT, UK \\ 2 Instituto de Astrofísica de Canarias, Calle Vía Láctea s/n, E38205, La Laguna, Tenerife, Spain \\ 3 Departamento de Astrofísica, Universidad de La Laguna (ULL), E-38206 La Laguna, Tenerife, Spain \\ 4 Centro de Astrobiología (CAB, INTA-CSIC), Carretera de Ajalvir km 4, E-28850 Torrejón de Ardoz, Madrid, Spain \\ 5 Departamento de Astrofísica, Facultad de CC. Físicas, Universidad Complutense de Madrid, E-28040 Madrid, Spain \\ 6 SISSA, via Bonomea 265, I-34136 Trieste, Italy \\ 7 Department of Physics, University of the Pacific, Stockton, CA 95211, USA \\ 8 Department of Physics and Astronomy, University of Pennsylvania, Philadelphia, PA 19104, USA
}

MNRAS, Accepted 2019 July 02. Received 2019 July 01; in original form 2018 December 10

\begin{abstract}
A diverse range of dust attenuation laws is found in star-forming galaxies. In particular, Tress et al. (2018) studied the SHARDS survey to constrain the NUV bump strength $(B)$ and the total-to-selective ratio $\left(R_{V}\right)$ of 1,753 star-forming galaxies in the GOODS-N field at $1.5<\mathrm{z}<3$. We revisit here this sample to assess the implications and possible causes of the correlation found between $R_{V}$ and $B$. The UVJ bicolour plot and main sequence of star formation are scrutinised to look for clues into the observed trend. The standard boundary between quiescent and star-forming galaxies is preserved when taking into account the wide range of attenuation parameters. However, an additional degeneracy - regarding the effective attenuation law - is added to the standard loci of star-forming galaxies in the UVJ diagram. A simple phenomenological model with an age-dependent extinction (at fixed dust composition) is compatible with the observed trend between $R_{V}$ and $B$, whereby the opacity decreases with the age of the populations, resulting in a weaker NUV bump when the overall attenuation is shallower (greyer). In addition, we compare the constraints obtained by the SHARDS sample with dust models from the literature, supporting a scenario where geometry could potentially drive the correlation between $R_{V}$ and $B$.
\end{abstract}

Key words: galaxies: ISM - ISM: dust, extinction - galaxies: stellar content

\section{INTRODUCTION}

Despite the fact that dust particles only account for less than 1 per cent in mass of the interstellar medium (Galliano et al. 2018), dust is a key component in galaxies. Starlight is affected in various ways depending on wavelength: it is scattered and absorbed, preferentially at shorter wavelengths and re-emitted in the infrared. For this reason, accurate dust corrections are fundamental when deriving stellar ages, stellar masses and star formation rates from photometry. By dust extinction, we refer both to the absorption and scattering of light away from the line of sight. Dust attenuation includes the contribution of the so-called 'star-dust geometry', where light from sources not along the line of sight may also

* E-mail: iferreras@iac.es scatter into it Calzetti 2001). A parameter typically used to characterize the attenuation law is the total-to-selective ratio $R_{V} \equiv A_{V} / E(B-V)$, where $E(B-V) \equiv A_{B}-A_{V}$ is the colour excess or reddening and $A_{X}$ is the extinction through the $X$ band. A lower value of $R_{V}$ implies a steep (strongly wavelength-dependent) attenuation law, whereas a higher value of $R_{V}$ produces a greyer (weakly dependent on wavelength) attenuation. In addition to the smooth dependence of the attenuation law with wavelength, a number of resonant features are present, most notably the $2,175 \AA$ bump in the NUV spectral window (Stecher 1969). This absorption feature is likely explained by small carbon-rich molecules, such as Polyaromatic Hydrocarbons (PAHs), or amorphous carbon (Galliano et al. 2018). It is present both in the extinction law of the Milky Way (MW) and the Large Magellanic Cloud (LMC), but absent in the metal poorer Small Magel- 
lanic Cloud (SMC, Pei 1992), although Hagen et al. (2017) report a milder NUV bump towards the north-east of the SMC.

Observations of individual stars in our Galaxy towards different sightlines reveal a diverse range of extinction laws (Cardelli, Clayton, \& Mathis 1989 Fitzpatrick \& Massa 2007), with similar results in nearby galaxies such as the LMC and the SMC (Gordon et al. 2003, Hagen et al. 2017. Galliano et al. 2018). For instance, in our Galaxy, we observe a wide range of $R_{V}$, varying from 2 to 5 , with an average of 3.1, adopted as the standard extinction law of the MW (see e.g. Fitzpatrick 1999). The non-universality of the dust extinction law is also seen in nearby galaxy M31 (Clayton et al. 2015). Tang et al. (2014, 2016) found that the more massive stars in resolved dwarf irregular galaxies are more reddened than less massive stars, indicating an age-selective extinction. Further afield, variations of the dust attenuation law (i.e. no longer considering single stars in the analysis) are found in the starburst galaxy M82, showing a radial gradient towards a steeper law and a weaker bump at large galactocentric distance (Hutton et al. 2015). Calzetti et al. I (1994, 2000) provide a comprehensive analysis of nearby starburst galaxies. The retrieved average attenuation curve is called the Calzetti law, frequently adopted to describe the effect of dust in starburst galaxies at all redshifts, and often applied to non starburst galaxies as well. This attenuation law lacks a bump and it is greyer $\left(\mathrm{R}_{V}=4.05\right)$ than that of the MW. Conroy et al. (2010) constrained the dust attenuation in a sample of low redshift galaxies combining GALEX and SDSS photometry, and found an overall weaker NUV bump with respect to our Galaxy. Wild et al. (2011) explored a large sample $(23,000)$ of star-forming galaxies, finding dust attenuation curves that were, on average, consistent with the presence of an NUV bump, with variations most likely influenced by the dust geometry. In contrast, the study of Battisti et al. (2016, 2017) found no confirmation of the NUV bump, with shallow attenuation curves. More recently, Salim et al. (2018) studied the dust attenuation law of more than 200,000 galaxies at low redshift. They observed a wide range of NUV bump strengths and dust attenuation slopes. In this study, steeper curves were found to correlate with the optical opacity, specifically higher opacities leading to a flatter curve.

Comparable results are obtained at higher redshift. For instance, Burgarella et al. (2005) conclude that their sample of UV-selected galaxies have dust attenuation curves similar to the LMC extinction law, but FIR-selected galaxies are similar to the MW curve. Noll et al. (2007) studied a sample of 108 massive star-forming galaxies at $1<z<2.5$. The NUV bump was present in a third of their sample. Galaxies with redder UV SEDs were associated with the presence of the carriers of the NUV bump. The study of $\mathrm{z} \sim 2$ galaxies of Buat et al. (2012) found that the UV dust attenuation increases with stellar mass and drops when the UV luminosity increases. Moreover, they detected the NUV bump in $20 \%$ of the galaxies. Additionally, Kriek \& Conroy (2013) used composite SEDs to explore the dust attenuation curve in a set of galaxies at $0.5<\mathrm{z}<2.0$. They found that the best-fit slope and the NUV bump strength are correlated, with steeper laws associated with a stronger bump. The Reddy et al. (2015) study on galaxies at $\mathrm{z} \sim 2$ found that, at shorter wavelengths, the dust curve has a similar shape as the Calzetti law, comparable to the SMC at $\lambda \gtrsim 2,500 \AA$. The study of Salmon et al. (2016) with galaxies at redshift $\mathrm{z} \sim 1.5-3$ found that a lower (higher) colour excess corresponds to a steeper (flatter) dust curve. However, they observe no correlation with other galaxy properties.

In general, studies provide evidence supporting the presence of a diverse range of attenuation properties (see,e.g. Battisti et al. 2017, Johnson et al. 2007, Wild et al. 2011 . Burgarella et al.|2005 | Buat et al. 2012 Conroy et al. 2010). In line with these studies, an analysis constraining the dust attenuation law at higher redshift was recently presented in Tress et al. (2018). The medium band $(\mathrm{R} \equiv \lambda / \Delta \lambda \sim 50)$, deep $(\lesssim 26.5 \mathrm{AB})$ optical photo-spectra of the Survey of High-z Absorption Red and Dead Sources (SHARDS, PérezGonzález et al. 2013) provides an optimal dataset to probe the dust attenuation law in the rest-frame NUV region of galaxies at redshift $\mathrm{z} \sim 2$. The results from this work agree qualitatively with Kriek \& Conroy (2013) and Salim et al. (2018), specifically a strong correlation is found between $R_{V}$ and $B$, whereby a steeper attenuation curve is associated with a stronger bump. A decreasing colour excess with increasing bump strength was also found.

Theoretical dust models allow us to account for the diversity of the dust attenuation laws. Charlot \& Fall (2000) strive to depict different effects of dust and its geometry. In this case, the younger population suffers not only the attenuation due to the presence of their own birth clouds but also the diffuse interstellar medium (ISM). Older stars, in turn, are affected only by the latter (e.g. Panuzzo et al. 2007). The study favours a mixed-slab model for the ISM. Along the same lines, Witt \& Gordon (2000) use multiple-scattering radiative transfer calculations to represent different types of galactic environment and dust composition (MW and SMC). Flatter dust curves with a weaker bump are found in clumpier dust distributions. Draine \& Li (2007) calculated the IR emission using a silicate-graphitePAH mixture dust model that agree with the extinction in the MW. More recently, Seon \& Draine (2016) produced a radiative transfer model in a spherical, clumpy interstellar medium (ISM). Radiative transfer effects cause weaker NUV bumps along with greyer attenuation curves when the ISM is clumpier and dustier. Additionally, a physical model for the presence of these variations in the dust attenuation law in galaxies was recently presented by Narayanan et al. (2018). They study cosmological hydrodynamic simulations of galaxy formation, implementing a 3D Monte Carlo dust radiative transfer code. They found that, even after assuming the same underlying dust composition, the attenuation laws rendered a wide range of properties. These variations are dependent on a diverse star-dust geometry. For instance, the different values of the NUV bump strength is associated to the fraction of unobscured $\mathrm{O}$ and $\mathrm{B}$ stars in their model.

The goal of this paper is to extend the analysis of the observed constraints on the dust attenuation law presented in Tress et al. (2018), specifically to study the correlation between $R_{V}$ and $B$, to shed light on the degeneracy between dust composition and the star-dust geometry, as potential drivers of this trend. The structure of the paper is as follows. In $\$ 2$ we reassess the SHARDS sample from Tress et al. (2018), exploiting its position on the UVJ bicolour diagram and on the main sequence of star formation in galaxies. $\$ 3$ presents a simple phenomenological model based on an 
age-dependent attenuation (at constant extinction, i.e. dust composition) that produces an effective attenuation law with similar properties to those observed in the SHARDS sample. 4 is devoted to a comparison of the observed trends with two dust models, namely Draine \& Li (2007) and Witt \& Gordon (2000). We conclude in \$5 with a discussion that wraps together the results obtained from the different tests performed in this paper.

\section{REVISITING THE SHARDS STAR-FORMING GALAXY SAMPLE}

This paper is motivated by a study of dust attenuation in a sample of star-forming galaxies compiled from SHARDS, the Survey for High-z Absorption Red and Dead Sources (Pérez-González et al. 2013). This study was presented in Tress et al. (2018). The SHARDS survey is an ultra deep galaxy survey towards the GOODS North field, comprising

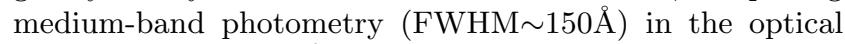

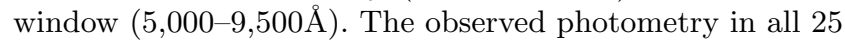
medium-band SHARDS passbands is combined with PSFmatched broadband photometry from HST in the optical and NIR (F435W, F105W, F125W, F140W, F160W) as well as Spitzer/IRAC $3.6 \mu \mathrm{m}$ fluxes and ground-based $\mathrm{K}_{s}$ data (see fig. 2 of Tress et al. 2018). The sample comprises 1,753 galaxies with high enough SNR $(\geqslant 5)$ across all SHARDS passbands, and cover the redshift window $1.5<\mathrm{z}<3$. The sample includes estimates of stellar mass, star formation rate and average, SSP-equivalent, stellar ages. The fluxes are compared with population synthesis models over a wide range of ages and chemical composition, and include dust attenuation as a foreground screen, following the generic law proposed by Conroy et al. (2010, hereafter CSB10). This law is fully defined by three free parameters: the total to selective extinction $\left(R_{V}\right)$, the NUV bump strength $(B)$, and the colour excess, $E(B-V)$. We note that the alternative parameterisation proposed by Noll et al. (2009) produces very similar results in our analysis. Appendix A of Tress et al. (2018) provides a simple functional mapping between these two choices. We follow a standard Bayesian approach, with flat priors on the parameters, to derive the a posteriori probability distribution function of the parameters, from which the best estimates and uncertainties are derived. See Tress et al. (2018) for more details about the fitting procedure, including comparisons with mock data following a wide range of star formation histories.

Our analysis of SHARDS star-forming galaxies revealed a wide range of attenuation laws. Most importantly, the dust-related parameters feature a significant correlation between $R_{V}$ and $B$ : as the dust attenuation law becomes flatter (higher $R_{V}$ ), the NUV bump weakens. This trend can have two possible causes. Firstly, as a result of a variation in the grain size distribution, the observed trend would imply that smaller dust grains are associated with a stronger NUV bump. A second explanation to this variation involves changes in the dust geometry among galaxies, i.e. the distribution of dust with respect to the underlying stellar populations.

This section extends the analysis of Tress et al. (2018), studying the distribution on the rest-frame UVJ colourcolour diagram, and seeking potential trends of the parame- ters with respect to the main sequence of star-forming galaxies.

\subsection{UVJ diagram}

We now turn our attention to the rest-frame UVJ colourcolour diagram. This diagram combines two different spectral intervals, and provides a relatively "cheap" diagnostic, in terms of telescope time, to disentangle the effects of dust from age (or metallicity). This technique is relatively widespread in the analysis of deep photometric data. A galaxy can appear red because the underlying stellar populations are old, therefore dominated by cool, evolved lowmass stars. However, a young, dust enshrouded galaxy could appear equally red, giving rise to the dust-age degeneracy. By contrasting the colour from a short interval in the region of the age-sensitive $4,000 \AA$ break $(U-V)$ with a wider one extending from the optical to the near infrared $(V-J)$, it is possible to discriminate between an old-dustless and a young-dusty galaxy. This diagram has been profusely used in the literature to classify galaxies into quiescent and starforming systems (e.g. Labbé et al. 2005. Williams et al. 2009, Whitaker et al. 2011, Cava et al. 2015, Domínguez Sánchez et al. 2016, Díaz-García et al. 2017).

However, the large variations found in the dust attenuation parameters could, in principle, affect the photometric data in ways that could alter the border between quiescent and star-forming galaxies. After all, the dust correction vector could shift and "put" some of the dusty star forming galaxies in the region of the UVJ diagram traditionally classified as quiescent - when adopting a fixed dust attenuation law. We studied this point by identifying our SHARDS sample on the UVJ plane (Fig. 1). We obtain the rest-frame $U-V$ and $V-J$ colours by use of the stellar population synthesis models of Bruzual \& Charlot (2003, hereafter BC03), adopting the best-fit stellar population and dust-related parameters. These best-fit values are obtained via a comparison of the SHARDS photometry as well as several NIR broadband fluxes with the BC03 models, including the generic dust extinction model of CSB10 (see Tress et al. 2018 , for details). We note the sample was selected by imposing a threshold in the SNR $(\geqslant 5)$ in all the SHARDS (optical) passbands of galaxies over the redshift range $1.5<\mathrm{z}<3$. This selection implies the galaxies have a high enough flux in the rest-frame NUV, meaning the sample should be made up of star-forming systems. As expected, the majority of our galaxies live on the star-forming region of the UVJ diagram, extending over a wide diagonal strip running from the bottom-left to the top-right, that mainly corresponds to the effect of dust attenuation on the colours.

The UVJ diagram in Fig. 1 is shown in several panels, dividing the sample with respect to redshift (left to right in increasing order of $\mathrm{z}$ ), colour coding the symbols with respect to each of the three dust-related parameters from top to bottom: $E(B-V) ; R_{V}$ and $B$. A typical error bar is shown on the leftmost panels (at the $1 \sigma$ level). The solid line limits the region of quiescent galaxies (to the top-left of the boundary), following the redshift-dependent definition of Whitaker et al. (2011). In general, the presence of the bump in a galaxy does not seem to largely affect its position within the UVJ diagram, although there is a preference towards stronger bumps in galaxies towards 


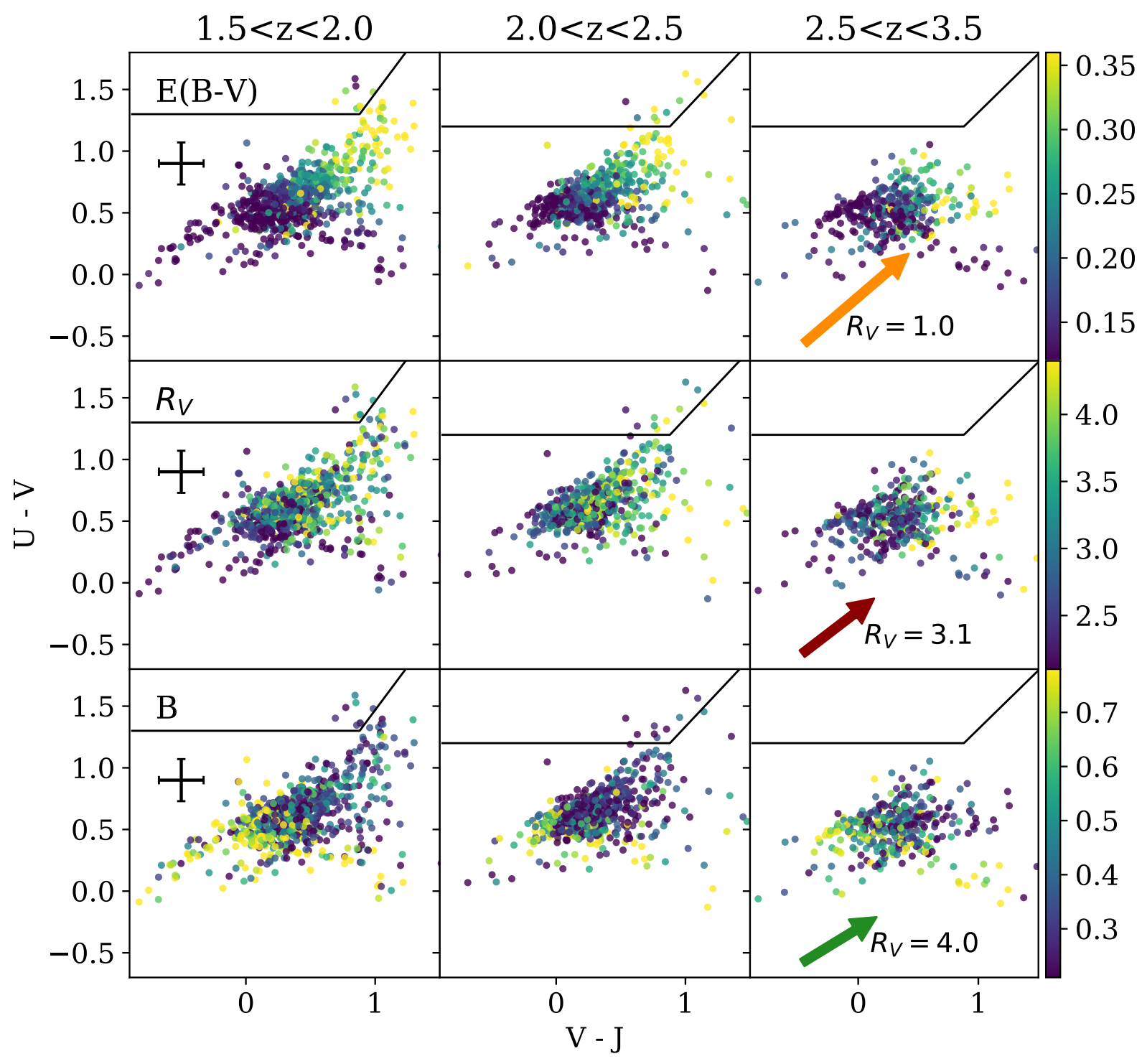

Figure 1. Rest-frame UVJ bicolour diagram of the SHARDS star forming sample from Tress et al. (2018). The distribution is colour coded with respect to the best-fit dust parameters, as labelled (from top to bottom, $E(B-V), R_{V}$ and $B$, with colour bars shown to the right in each row). The sample is shown in three bins of redshift (increasing from left to right), and we include the boundary between quiescent and star-forming galaxies, as defined in Whitaker et al. (2011). The arrows on the rightmost panels give the dust correction at $A_{V}=1$ at three different values of $R_{V}=\{1.0,3.1,4.0\}$, as labelled.

the blue-blue (i.e. bottom-left) part of the diagram. The total-to-selective ratio also shows a trend, with steeper (i.e. lower $\mathrm{R}_{V}$ ) attenuation towards the same part of the diagram. As expected, the colour excess, $E(B-V)$, shows the strongest trend, with higher values towards the top-right part of the diagram. To quantify these trends, we calculated the Kolmogorov-Smirnov (KS) statistic, extended to twodimensional datasets, as implemented by Peacock (1983) and Fasano \& Franceschini (1987). In each case, we split the sample at the median value of the given dust parameter, and compare the resulting samples with the KS test.
We find a KS statistic $D_{\mathrm{KS}}$ of 0.54 (when splitting the sample with respect to $E(B-V)$ ); 0.32 (for a $R_{V}$ split); 0.30 (for a $B$ split). To assess the significance of these values, we performed 1,000 random splits of the sample (i.e. creating subsets with the same number of galaxies) producing a Monte Carlo distribution that gave $D_{\mathrm{KS}}=0.06 \pm 0.01(1 \sigma)$, confirming that the trends with respect to the dust related parameters are statistically significant, with the most significant one being the split in colour excess.

At the highest redshift bin (rightmost panels), we observe comparatively fewer red galaxies. This may be ex- 


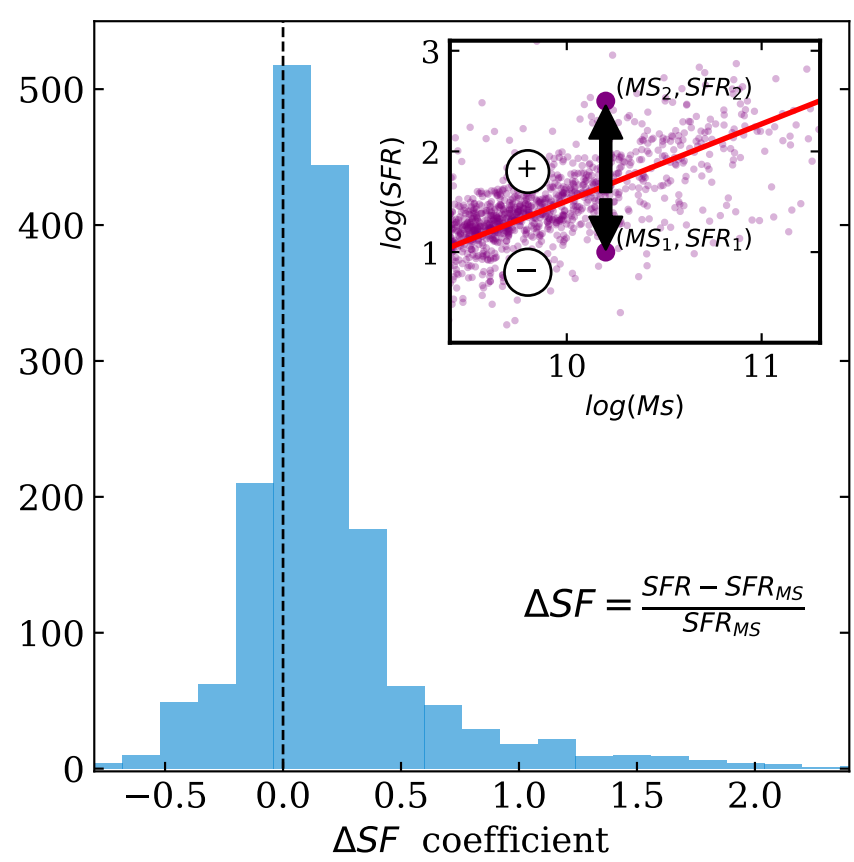

Figure 2. Distribution of the $\Delta S F$ parameter of our SHARDS sample. The definition of this parameter is illustrated in the inset. $\triangle S F$ has a positive or negative value depending on the position of the data point with respect to the Main Sequence (MS) defined on the plane spanned by $\log$ SFR vs $\log M_{s}$.

plained by an observational bias imposed by the flux and SNR limit of the sample selection. The rightmost panel of Fig. 1 illustrates the effect of a varying dust attenuation law by showing three dust vectors for $A_{V}=1$, corresponding to, from top to bottom: $R_{V}=\{1.0,3.1,4.0\}$, as labelled, assuming a Milky Way NUV bump strength $(B=1)$. Note how a steeper dust attenuation law (lower $R_{V}$ ) results in a higher reddening at fixed $A_{V}$ - as expected from the definition of colour excess: $E(B-V) \equiv A_{V} / R_{V}$. This figure confirms that the wide range of dust attenuation values does not affect the separation between quiescent and star-forming galaxies, as those variations follow the same direction as changes in dust. However, a new degeneracy is added in the analysis, so that the diagonal strip of star-forming galaxies span a range of age, metallicity, colour excess and $R_{V}$.

\subsection{Main sequence (MS) of star formation}

In order to characterise further our galaxy sample, we explore the correlation between the derived star formation rate (SFR) and stellar mass. In particular, we want to compare the activity with respect to the Main Sequence (MS) of star forming galaxies. We adopt the definition of the MS given by Speagle et al. (2014). It is important to note that the sample used for this particular exercise corresponds to objects with well-defined SFRs (i.e. those galaxies that only have upper limits are excluded here). In order to parameterise the location of a galaxy on the SFR vs mass diagram with respect to the MS, we define a dimensionless coefficient $\Delta S F$. This coefficient represents the fractional offset in the SFR between the targeted galaxy and the Main Sequence, at fixed mass and at the redshift of the galaxy, namely:

$$
\Delta S F(z)=\frac{S F R-S F R_{M S z}}{S F R_{M S z}},
$$

where, $S F R_{M S z}$ is the star formation rate at the position of the MS for the stellar mass and redshift as the targeted galaxy. The star formation rates of our sample are taken from Wuyts et al. (2011), who use photo-spectra covering a wide range from the $U$ band to $8 \mu \mathrm{m}$ fluxes. We use their SFR $_{U V+I R}$ indicator, and note that all our SHARDS sources have star formation rate estimates. As a check on the effect of redshift on the derivation of $\Delta S F$, we compare the analysis using the MS at the median redshift $\mathrm{z}=2$, and then recalculated $\triangle S F$ taking the redshift-dependent MS. The differences between these two methods are negligible. Fig. 2 shows the distribution of the $\triangle S F$ coefficient. A positive (negative) value means the observed SFR is higher (lower) than the one the galaxy should have if it were on the Main Sequence. Most of our sample falls close to the MS relation, with variations mostly within $\pm 35 \%$ (over $77 \%$ of the sample has $|\Delta \mathrm{SF}| \leqslant 0.35$ ). The inset of Fig. 2 illustrates the definition of this coefficient. We refer the reader to fig. 1 of Tress et al. (2018) for a standard representation of our SHARDS sample with respect to the MS.

Fig. 3 shows the distribution of $\triangle S F$ with respect to the three dust-related parameters (from top to bottom) $E$ ( $B-$ $V), R_{V}$ and $B$. In all panels, the lines (and shade) trace the median (and $1 \sigma$ scatter) of subsamples, split with respect to stellar age (blue-solid: young tercile, red-dashed: old tercile). The median stellar age of the distribution is 5.2 Myr (note, for reference, the plots regarding the distribution of other observables of the sample in fig. 7 of Tress et al. 2018). Our sample is slightly biased towards stronger star formation than MS galaxies, with a median of $\Delta \mathrm{SF}_{m}=+0.10$, with the offset dominated by younger galaxies (the median for this subsample, represented by the blue shaded regions in Fig. 3 is +0.17 ). Consistently with the findings in Tress et al. (2018), we recover the trend towards higher values of $R_{V}$ for the older population, and a higher colour excess in younger star-forming galaxies. Moreover, the figure suggests a weak decrease of the NUV bump with increasing $\Delta \mathrm{SF}$ in the young subsample. However, given the typical error bars expected in the derivation of $B$ (around 0.2 , see table 2 of Tress et al. 2018), we can only present this as a marginal trend.

\section{AGE-DEPENDENT EXTINCTION: A PHENOMENOLOGICAL MODEL}

The standard approach to study the attenuation law and its relation to the dust composition and its distribution among the stellar populations involves radiative transfer modelling (see, e.g., Witt \& Gordon 2000 Groves, Dopita, \& Sutherland 2004 | Panuzzo et al. 2007|| Popescu et al. 2011|| Natale et al. 2015, Seon \& Draine 2016). However, a simple phenomenological model can be explored, that encapsulates the fundamental aspect dealing with the effect of the distribution of dust within the stellar populations. We present such model here. We make the working hypothesis that the extinction law (i.e. the wavelength dependence of dust scattering and absorption, dependent on the dust composition) 


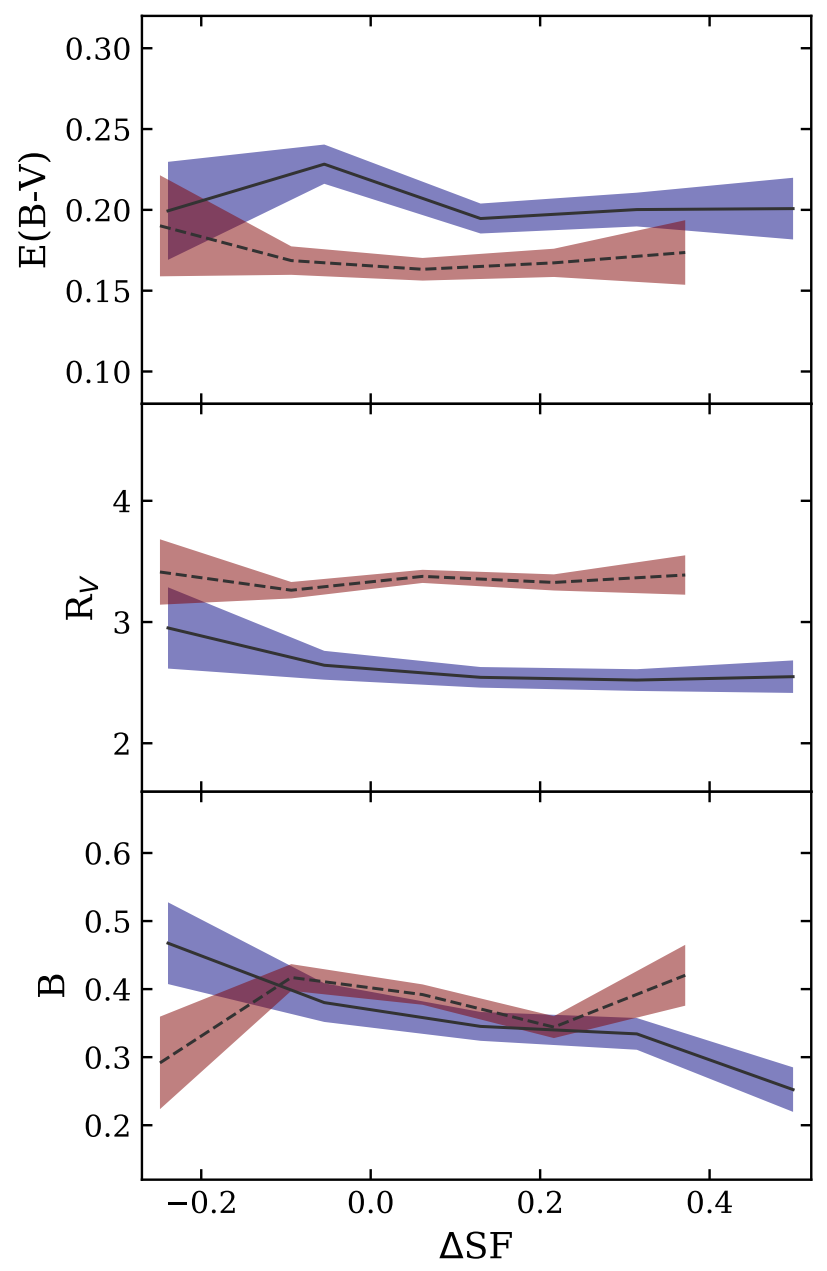

Figure 3. Comparison of the $\Delta \mathrm{SF}$ coefficient (see Fig. 2) with the best-fit dust-related parameters. In all panels, the lines (and shade) trace the median (and $1 \sigma$ scatter) of subsamples, split with respect to stellar age (blue-solid: young tercile, red-dashed: old tercile). The median stellar age of the distribution is 5.2 Myr.

is the same in all galaxies, whereas the observed variations in the attenuation law among galaxies are produced as a result of a different "geometry". Dust is expected to mostly affect the youngest populations, typically found in gas-rich and dust-rich regions. As age progresses, these populations - along with the gas and dust content - disperse, resulting in weaker attenuation. Silva et al. (1998) presented for the first time a galaxy model with an age-selective and geometry dependent extinction. Similarly, Charlot \& Fall (2000) proposed a simple description of attenuation in galaxies involving two different dust laws: a diffuse one for old populations and a higher opacity relation for the young components that are still embedded in very dusty environments.

We extend this model here, adding a smooth time dependence, in the sense that the overall level of extinction parameterised by the colour excess, $E(B-V)$ - for a given, single-age, population decreases monotonically with time. We assume a constant star formation rate for this model. The extinction for each component (and, thus, the dust grain composition) is kept fixed at the standard Milky Way law (i.e. $R_{V}=3.1$ and $B=1$, adopting the functional form of
CSB10). Two functions are considered for the time dependence: a linear function with (log) time, or an exponentially decreasing function. In either case, a single parameter quantifies the timescale over which the effect of dust decreases. We emphasize that this is a strong assumption meant to assess whether an average composition of Milky Way-like dust could lead to attenuation trends qualitatively similar to the observations. We also note that the timescales considered here are significantly longer than those adopted in the birth cloud model of Charlot \& Fall (2000). Our model does not aim to follow the physical processes in single star-forming clouds - within which dust dispersion and destruction operate over significantly shorter timescales - but, rather, to define a phenomenological parameter that takes into account the global evolution of the dust content over galaxy scales. Therefore, this timescale includes the complex aspects of the star formation history and the evolution of dust geometry. Detailed radiative transfer models, beyond the scope of this paper, would be required to relate our longer timescales with the shorter ones typically used in the birth cloud model.

\subsection{Linear decrease of reddening}

We build a set of simulated data by combining the BC03 stellar population synthesis models and adopting an agedependent dust attenuation. We enforce the same distribution of redshifts, flux uncertainties and SHARDS passband offsets as the original SHARDS sample presented in Tress et al. (2018). We impose the following dependence for the colour excess:

$$
E(B-V)(t)=\max \left[\epsilon_{M}-\mu \log \left(\frac{t}{0.01 \mathrm{Gyr}}\right), 0\right],
$$

where the composite stellar population follows a constant star formation rate between 0.01 and 1 Gyr. The normalization of this expression is given by $\epsilon_{M}$, i.e. the maximum reddening suffered by the population. In the simulated data, $\epsilon_{M}$ is extracted from a uniform random deviate between 0.1 and 0.6 , and $\mu$ is chosen so that the amount of reddening at $\mathrm{t}=1$ Gyr is zero. Following this model, a higher $\mu$ produces a rapidly changing extinction, as if the dust were dispersed very efficiently. Although this is admittedly a very simple phenomenological model, our aim is to assess whether an age-dependent dust extinction model could lead to the correlation found between the dust-related parameters.

Once the mock fluxes are produced, we subject the data to the same analysis pipeline as the one presented in Tress et al. (2018), using exactly the same SSP model grids. The results are shown in Fig. 4 , presenting the trends on the $R_{V}$ vs $B$ plane. The results are colour coded according to $\mu$. It is important to note that, by construction, all data feature the same intrinsic (Milky Way) extinction, represented by a star symbol in the plot. The figure shows that the introduction of an age-dependent extinction - acting as a proxy for the dust geometry - induces a correlation between the dust parameters of the effective attenuation law. The resulting trend (the solid red line is the result of a linear regression to the data) agrees qualitatively with the observational result from Tress et al. (2018), i.e. a stronger bump is expected when the attenuation law is steeper (lower $\mathrm{R}_{V}$ ). The dotted line shows the observed trend from that work. A systematic 


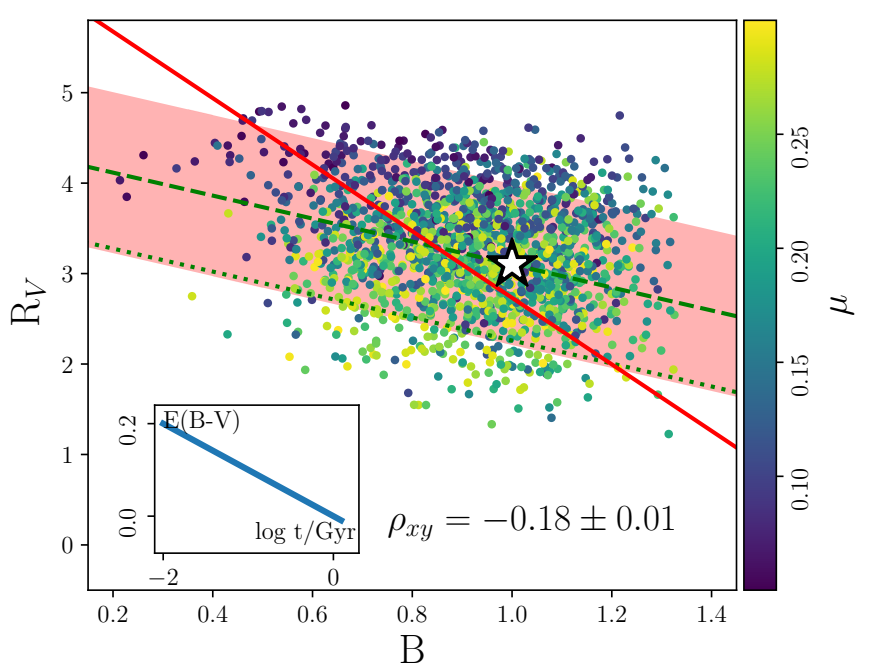

Figure 4. Relationship between $R_{V}$ and $B$ when modelling an age-dependent attenuation that varies linearly with (log) time, parameterised by a slope $\mu$ (equation 2 see inset for an example). The dots are colour coded according to the value of $\mu$ (colour bar on the right). The red solid line shows the best-fit, and the linear correlation coefficient $\left(\rho_{x y}\right)$ is included (along with a $1 \sigma$ error bar). The star symbol marks the standard extinction law of the Milky Way. The dashed line and shaded area show the observed slope and scatter from Tress et al. (2018) when imposing the Milky Way constraint at $B=1$, and the dotted line is the original relation.

can be expected from a range of factors, including variations in dust composition, or a more detailed description of the age-dependent attenuation. To aid the eye, we shifted the observed trend, imposing the constraint from the Milky Way extinction curve, so that $R_{V}=3.1$ at $B=1$, shown as a dashed line, with a shaded area representing the observed scatter in the trend.

Galaxies with a lower value of $\mu$ (slower time variation) tend to have a greyer attenuation curve. Galaxies with a stronger NUV bump have an attenuation curve comparable to the Milky Way, although there is a wide scatter. We note that the parameter uncertainty expected from this methodology is $\Delta R_{V} \sim 0.7$ and $\Delta B \sim 0.2$ (see Tab. 2 in Tress et al. 2018). To quantify the trend introduced by the time variation, we computed the linear correlation coefficient between $\mathrm{B}$ and $\mathrm{R}_{V}$, obtaining ${ }^{1} \rho_{x y}=-0.18 \pm 0.01$. The error bar $(1 \sigma)$ is obtained from a Monte Carlo analysis comprising 100 realizations produced by adding noise to all data points corresponding to the error bars of the individual parameter estimates. Therefore, the trend is robust. We emphasize that this exercise is not meant to fully explain the observational trends, but to motivate how a fixed extinction law could produce the observed correlations if the dust were to affect the stellar populations in a time-dependent way. Variations in the intrinsic dust extinction law - caused by changes in the dust composition - will potentially induce changes that can explain the mismatch between this phenomenological model and the observational trends.

1 We follow the standard notation, $\rho_{x y}$ for the linear correlation coefficient. In this case $\mathrm{x}=B$ and $\mathrm{y}=\mathrm{R}_{V}$.
This model is qualitatively similar to the 'birth model' of Charlot \& Fall (2000), with younger stars being more attenuated due to the presence of their birth clouds, and later populations being progressively less attenuated as the dust is being dispersed or destroyed. However, we note that the timescales involved in our model are substantially longer: the classical model assumes that after $\sim 10 \mathrm{Myr}$, the stars break out of the birth cloud. However, we note that our phenomenological timescale also incorporates the effect of additional processes related to the evolution of dust over galaxy scales. Regarding the variation of the $B$ parameter, Panuzzo et al. (2007) predict that young obscured stars have a weaker bump. Following this exercise, since the underlying extinction remains the same, we may argue that geometry plays a fundamental role in the observed trends presented in Tress et al. (2018).

\subsection{Exponential decrease of reddening}

An additional test of the age-dependent extinction scenario is to assess how the functional form of this dependence affects the derived effective attenuation. We decided to explore an exponentially decaying law, where the colour excess is described by:

$$
E(B-V)(t)=\epsilon_{M} e^{-t / \tau},
$$

and $\tau$ introduces a timescale, representing the efficiency of disintegration/dispersal of dust. The rate of change with time is slowed in this model, with respect to the linear model with (log) time, for the range of parameters explored. Note as $\tau \rightarrow 0$, we concentrate the dust only on the very youngest phases of star formation, whereas $\tau \rightarrow \infty$ represents a foreground dust screen (as all populations are equally affected by the same level of extinction). To create the mock data we follow a similar methodology as in the linear case regarding the distribution of redshift, flux uncertainty and passband offsets, as well as the underlying (constant) star formation history between 0.1 and 1 Gyr. This model has two free parameters: for each galaxy a random pair $\left(\tau, \epsilon_{M}\right)$ is chosen from a uniform random deviate, between $\tau=0.1$ and $1 \mathrm{Gyr}$ and between $\epsilon_{M}=0.1$ and $0.6 \mathrm{mag}$. The synthetic data are analysed in the same way as the observed SHARDS starforming galaxies of Tress et al. (2018). Fig. 5 shows the trend of the dust parameters on the $R_{V}$ vs $B$ plane.

A similar correlation is found between $R_{V}$ and bump strength (blue solid line) as in the previous case, in qualitative agreement with the observed trend, given by a dotted line. Analogously to the previous case, we shift the trend by a constant value, using the Milky Way constraint at $B=1$, represented by the dashed line and the shaded area. The data suggest higher values of $\tau$ (i.e. a weaker age-dependent extinction) produce a greyer attenuation law. The linear correlation coefficient is $\rho_{x y}=-0.15 \pm 0.01$. Therefore, at face value, this simple exercise suggests that an age-dependent extinction provides a suitable description of the destruction and dispersal of dust in star-forming galaxies, and can explain - at least in part - the observed anticorrelation between $R_{V}$ and bump strength. 


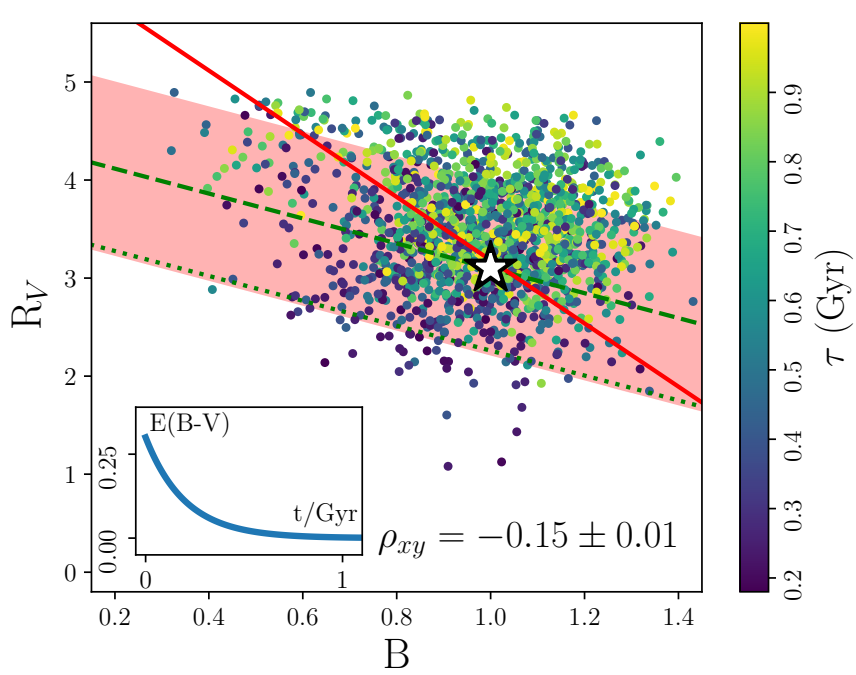

Figure 5. Equivalent to Fig. 4 for a model where the colour excess changes exponentially with time, given by a free parameter $\tau$ (equation 3 see an example in the inset). The star symbol marks the standard extinction law of the Milky Way. The dots are colour coded according to the value of $\tau$ (colour bar on the right). The red solid line shows the best-fit. The linear correlation coefficient $\left(\rho_{x y}\right)$ is included along with a $1 \sigma$ error bar. The dashed line and shaded area show the observed slope and scatter from Tress et al. (2018) when imposing the Milky Way constraint at $B=1$, and the dotted line is the original relation.

\section{COMPARISON WITH STANDARD DUST ATTENUATION MODELS}

In addition to the phenomenological age-dependent extinction model shown above, we compared the results of our SHARDS star-forming galaxies with two of the standard dust attenuation models, Draine \& Li (2007) and Witt \& Gordon (2000).

\subsection{Draine \& Li models}

The models of Draine \& Li (2007) provide a way to derive fundamental properties of the dust component from infrared observations with Spitzer's IRAC and MIPS cameras. These models consist of a mixture of Polyaromatic Hydrocarbons (PAHs), carbonaceous grains, and amorphous silicate grains, heated by starlight. The main parameters are the mass fraction of the dust content in the form of PAHs $\left(q_{\mathrm{PAH}}\right.$, usually given as a percentage), the lower cutoff of the starlight intensity distribution $\left(U_{\min }\right)$, the fraction of dust mass $(\gamma)$ that is exposed to starlight intensities $U_{\min }<U \leqslant U_{\max }$, and the total dust mass $\left(M_{d}\right)$. The full set of SHARDS fluxes, combined with Spitzer and Herschel data (Pérez-González et al. 2013) was used to derive these parameters following the prescriptions set out in Draine \& Li (2007). The parameters are fitted to galaxies with $24 \mu \mathrm{m}$ fluxes plus, at least, one flux data point from Herschel, needed to lift the degeneracy between the model parameters. Only galaxies with IR fluxes having $\mathrm{S} / \mathrm{N} \geqslant 3$ are considered in this analysis. Milky Way type dust was assumed. Given the very discrete values of the dust model parameters published by Draine \& Li (2007), we interpolated them to obtain more models with a finer parameter resolution in $\gamma$ and $q_{\mathrm{PAH}}$. The fitting procedure compares the data from the model grid to the observed fluxes for each galaxy, following a standard $\chi^{2}$ statistic, so that the best-fit parameters correspond to the minimum value of the statistic. A Monte Carlo method is adopted, where the fitting procedure is repeated 10 times per galaxy, adding in each realization a Gaussian random deviate to the observed fluxes with zero mean and variance equivalent to the flux uncertainties. The distribution of best fit values for each galaxy allows us to derive the uncertainty of the parameters. We note that these parameters are constrained with infrared fluxes, therefore requiring mostly independent information with respect to the dust parameters presented in Tress et al. (2018), that focus on the rest-frame NUV and optical windows. There is a total of 71 galaxies with good fits to these models. We note that the redshift distribution of this subsample is undistinguisable from the original dataset, but the sample is biased towards the massive end. The median and standard deviation of the stellar mass is $\log M_{s} / M_{\odot}=10.53 \pm 0.55$ in contrast with $9.59 \pm 0.56$ for the original sample.

Figure 6 shows the constraints to the Draine \& $\mathrm{Li}$ model parameters with respect to our dust-related parameters (from left to right): $R_{V}, B$ and $E(B-V)$. Individual data points are shown as grey dots, whereas the solid lines follow the median of binned data at a fixed number of data points per bin. The vertical bars in the lines represent the median uncertainty at a $1 \sigma$ level. A characteristic error bar is included in each panel, corresponding to the median value of the $1 \sigma$ uncertainty of the relevant parameters. The $\mathrm{PAH}$ fraction $\left(q_{\mathrm{PAH}}\right)$ increases with $B$ and colour excess, suggesting that dustier environments with a prominent NUV bump feature a higher fraction of such molecules. It is commonly accepted that graphite-like, very small dust particles are the carrier of the NUV bump (e.g. Draine 1989). The fraction of dust exposed to starlight $(\gamma)$, within the parameterised range of intensity, is found to decrease with increasing colour excess, and possibly (although weakly so) $\mathrm{R}_{V}$, potentially as a consequence of shielding in dustier environments. The trends of dust mass are rather weak, with prominent scatter, and a hint of an increasing trend with colour excess. Finally, the starlight intensity distribution $U_{\min }$ is found to decrease with increasing $E(B-V)$, and also to decrease as the NUV bump becomes less prominent. However, we emphasize that these trends should be considered semi-quantitative, as the range of variation and the underlying uncertainties are rather large. More detailed modelling would be necessary, but is beyond the scope of this paper.

\subsection{Witt \& Gordon dust models}

One of the standard benchmarks to characterize the attenuation law in galaxies is provided by the dust models of Witt \& Gordon (2000, hereafter WG00). The models explore three different galactic environments: CLOUDY, DUSTY and SHELL. Each one features a different distribution of dust with respect to the underlying stellar component. Specifically, SHELL corresponds to a stellar region surrounded by a dust shell; DUSTY has a uniform mixture of stars and dust and CLOUDY is defined by a mixed duststellar region surrounded by a dust-free region. In addition to the global dust geometry, these models also adopt two 

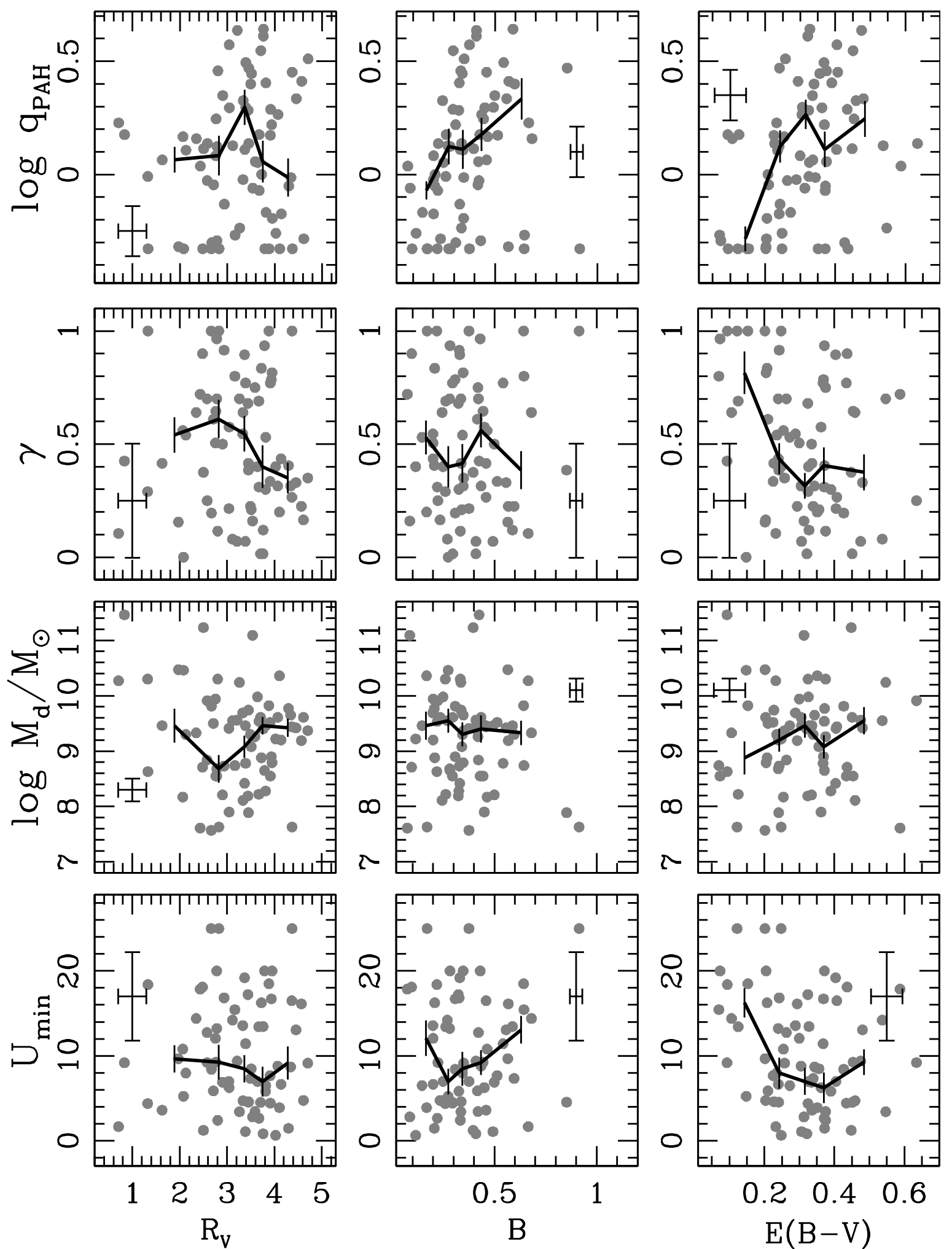

Figure 6. Distribution of dust parameters following the models of Draine \& Li (2007), from top to bottom the PAH strength (log $\left.q_{\mathrm{PAH}}\right)$, the fraction of dust mass $(\gamma)$ that is exposed to starlight intensities $U_{\min }<U \leqslant U_{\max }$, the dust mass (log $M_{d}$, in solar units), and the radiation field threshold $\left(U_{\min }\right)$. A running median, including vertical lines to represent the RMS scatter within each bin, is shown as a solid line. The error bar in each panel gives a representative (median) $1 \sigma$ uncertainty of the parameter estimates, from a Monte Carlo run (see text for details). 
different types of local structure: homogeneous and clumpy. There are significant variations between these two. For instance, the NUV bump strength is weaker in the clumpy case, since it has a greyer wavelength dependence of the attenuation, with respect to the homogeneous structure (Witt \& Gordon 2000). WG00 models adopt two different intrinsic extinction prescriptions, corresponding to the Milky Way and the bar of the Small Magellanic Cloud. The variations among these diverse scenarios concern the different return of scattered radiation, producing a range of effective attenuation laws. For instance, the homogeneous SHELL model is comparable to a spherical homogeneous screen with flux scattered back into the beam (Witt \& Gordon 2000).

We compare the functional dependence of the CSB10 dust attenuation function (i.e. the $B$ and $R_{V}$ parameterisation) with respect to the WG00 models. Moreover, we include the equivalent parameters $E_{b}$ and $\delta$ in the notation of Noll et al. (2009). This alternative parameterisation defines the NUV bump as a Lorentzian-like Drude profile (similarly but not equivalently to CSB10, so that parameters $B$ are $E_{b}$ are related, but are not identical), and describes the varying total-to-selective ratio as a multiplicative power law $\propto \lambda^{\delta}$ applied to a Calzetti-like dust law (so that $\delta=0$ corresponds to $\mathrm{R}_{V}=4.05$, see Noll et al. 2009 for details). A translation between these two choices of the attenuation law can be found in Appendix A of Tress et al. (2018).

We consider three optical depths, namely $\tau_{V}=$ $\{0.5,1.5,3.0\}$. The comparison - within the spectral window $\lambda \in[1300,7500] \AA$ - is done with a PYTHON script that fits the publicly available WG00 attenuation curves with these models, following a two stage process, starting with a Sequential Least SQuares Programming ${ }^{2}$ fit with a large error bar (assuming 20\% of the value of the opacity at each wavelength), followed by an MCMC search starting with the best-fit value using the EMCEE sampler (Foreman-Mackey et al. 2013), reducing in this second stage the error bars to a $5 \%$ level. The output after 400 steps (removing the first 100 burn-in steps) produces a distribution from which the median and RMS of each parameter is obtained, shown in Tab. 1. The final result - taking the median of the distribution for each parameter as best fit - is plotted and visually inspected to confirm the convergence of the fitting procedure. In general, a slightly steeper dust attenuation curve is found in the homogeneous case, in comparison to the clumpy scenario. As the optical depth increases, the NUV bump strength decreases for both CLOUDY and DUSTY geometries. This behaviour is observed both in the homogeneous and clumpy cases. In particular, in the CLOUDY scenario, shifting from a moderate optical depth to a very optically thick case results on the strength of the NUV bump to be halved. The same occurs in a clumpy SHELL geometry. This is a trend observed in galaxies at low redshift, where the NUV bump is stronger at lower optical opacities (Salim et al. 2018). On the other hand, for the homogeneous case there is no significant change regarding $B$. In contrast, the total-to-selective ratio $R_{V}$ increases in every case, although for the SHELL homogeneous scenario, this increase is less obvious. As the optical depth increases, the attenuation be2 calling SciPy function optimize.minimize with method
SLSQP. comes greyer, a trend reported in several samples (Salmon et al. 2016, Tress et al. 2018, Salim et al. 2018, Narayanan et al. 2018).

Fig. 7 compares the clumpy version of the WG00 models to the observational constraints to the attenuation law of the SHARDS star-forming galaxies from Tress et al. (2018), showing the individual galaxies as grey dots and the solid red line tracing the best fit to the models. The star locates the fiducial Milky Way extinction law. The WG00 models do not provide a good match to the data, mainly as the best-fit values of $R_{V}$ are too high, producing substantially greyer models, and also rather prominent NUV bump strengths. However, the trends have similar slopes, from a steeper law with a strong bump (at low opacity) towards a weaker bump with a greyer attenuation. We note that the WG00 models explored here adopt the Milky Way extinction law. We did not fit the models with an SMC-type extinction, since, by definition, the CSB10 function is parametrised taking the Milky Way extinction law as a reference. Moreover, the lack of a bump in the extinction law of the SMC would result in a trivial $B=0$ parameter in all cases.

\section{DISCUSSION}

This paper extends the analysis of the sample of high redshift, star-forming galaxies presented in Tress et al. (2018). In that paper, we exploited the SHARDS survey (PérezGonzález et al. 2013) to constrain the dust attenuation law of star-forming galaxies over the redshift interval $1.5<\mathrm{z}<3$. A substantial correlation was found between the total-toselective extinction (parameterised by $R_{V}$ ), the strength of the NUV bump, and the colour excess. The interpretation involves two main aspects of dust in galaxies: its intrinsic composition (affecting the extinction law) and the distribution of dust within the underlying stellar components (the so-called "geometry"). We explore in this paper an eclectic set of potential ramifications of the wide range of dust attenuation parameters found in star-forming galaxies, mostly related to diagnostics of star formation vs quiescence or to the interpretation of the observed variations in light of a few well established models of dust attenuation.

Our first aim concerns the characterisation of the sample of SHARDS galaxies regarding star formation activity, by putting them on a rest-frame UVJ colour-colour diagram. Such diagrams are typically used to classify galaxies as either quiescent or star-forming systems. The wide range of dust attenuation parameters found in Tress et al. (2018) raised the issue whether the standard classification based on this bicolour plot could be affected by it. Our analysis showed that the loci used to define quiescent galaxies was not compromised as the observed variations followed the same direction on the colour-colour diagram as the overall extinction $A_{V}$ (Fig. 1). However, these variations introduce a more complex degeneracy along the diagonal direction of the diagram with respect to age, metallicity, colour excess and total-to-selective extinction $\left(R_{V}\right)$. The strength of the NUV bump is also found to correlate with the location of the galaxies on the UVJ plane (note that this feature is rather wide, so it can affect the fluxes through the $U$ passband). We also assessed a potential correlation of the dustparameters with respect to the location of these galaxies 
Table 1. Fit of the Witt \& Gordon (2000) models (Milky Way extinction) to the generic attenuation function of Conroy et al. (2010).

\begin{tabular}{|c|c|c|c|c|c|c|c|c|}
\hline$\tau_{V}$ & $B$ & $R_{V}$ & $E_{b}$ & $\delta$ & $B$ & $R_{V}$ & $E_{b}$ & $\delta$ \\
\hline \multicolumn{9}{|c|}{ CLOUDY } \\
\hline & \multicolumn{4}{|c|}{ homogeneous } & \multicolumn{4}{|c|}{ clumpy } \\
\hline 0.5 & $1.46 \pm 0.10$ & $3.77 \pm 0.07$ & $5.89 \pm 0.46$ & $+0.13 \pm 0.01$ & $1.32 \pm 0.10$ & $3.95 \pm 0.07$ & $5.04 \pm 0.43$ & $+0.17 \pm 0.01$ \\
\hline 1.5 & $0.91 \pm 0.09$ & $4.61 \pm 0.08$ & $2.77 \pm 0.37$ & $+0.31 \pm 0.01$ & $0.80 \pm 0.09$ & $4.95 \pm 0.09$ & $2.24 \pm 0.35$ & $+0.38 \pm 0.01$ \\
\hline 3.0 & $0.59 \pm 0.09$ & $5.53 \pm 0.09$ & $1.37 \pm 0.33$ & $+0.49 \pm 0.01$ & $0.51 \pm 0.09$ & $5.78 \pm 0.10$ & $1.14 \pm 0.32$ & $+0.54 \pm 0.01$ \\
\hline \multicolumn{9}{|c|}{ DUSTY } \\
\hline & \multicolumn{4}{|c|}{ homogeneous } & \multicolumn{4}{|c|}{ clumpy } \\
\hline 0.5 & $1.69 \pm 0.11$ & $3.50 \pm 0.06$ & $7.38 \pm 0.51$ & $+0.06 \pm 0.01$ & $1.54 \pm 0.11$ & $3.64 \pm 0.06$ & $6.46 \pm 0.48$ & $+0.10 \pm 0.01$ \\
\hline 1.5 & $1.33 \pm 0.10$ & $3.85 \pm 0.07$ & $5.16 \pm 0.44$ & $+0.15 \pm 0.01$ & $1.12 \pm 0.10$ & $4.25 \pm 0.07$ & $3.87 \pm 0.40$ & $+0.24 \pm 0.01$ \\
\hline 3.0 & $1.08 \pm 0.10$ & $4.38 \pm 0.07$ & $3.62 \pm 0.40$ & $+0.26 \pm 0.01$ & $0.83 \pm 0.09$ & $4.94 \pm 0.08$ & $2.34 \pm 0.36$ & $+0.38 \pm 0.01$ \\
\hline \multicolumn{9}{|c|}{ SHELL } \\
\hline & \multicolumn{4}{|c|}{ homogeneous } & \multicolumn{4}{|c|}{ clumpy } \\
\hline 0.5 & $1.84 \pm 0.11$ & $3.28 \pm 0.06$ & $8.61 \pm 0.53$ & $+0.01 \pm 0.01$ & $1.60 \pm 0.11$ & $3.54 \pm 0.06$ & $6.87 \pm 0.49$ & $+0.08 \pm 0.01$ \\
\hline 1.5 & $1.74 \pm 0.10$ & $3.22 \pm 0.05$ & $8.17 \pm 0.52$ & $-0.00 \pm 0.01$ & $1.17 \pm 0.10$ & $4.03 \pm 0.07$ & $4.27 \pm 0.41$ & $+0.19 \pm 0.01$ \\
\hline 3.0 & $1.65 \pm 0.11$ & $3.23 \pm 0.05$ & $7.69 \pm 0.52$ & $-0.00 \pm 0.01$ & $0.87 \pm 0.09$ & $4.73 \pm 0.08$ & $2.58 \pm 0.37$ & $+0.33 \pm 0.01$ \\
\hline
\end{tabular}

relative to the main sequence of star formation. Most of our sample falls close to the main sequence, with variations within the $25 \%$ level (Fig. 2), with no statistically significant correlation with the dust parameters (Fig. 3).

We explored the efficiency of dust destruction/dispersal with a simple phenomenological model given by an agedependent reddening. We used two different efficiencies, a linear decrease of reddening with $(\log )$ time (Fig. 4); and an exponential decrease (Fig. 5). Both scenarios are extensions of the 'birth cloud' model over longer timescales, whereby younger stars suffer more dust attenuation compared to older stars. The timescale of our phenomenological model includes the contribution from additional mechanisms that regulate the global evolution of dust over galaxy scales. We built mock data from the Bruzual \& Charlot (2003) population synthesis models, applying an age-dependent attenuation with a fixed (Milky Way standard) extinction, as a test of possible causes of the correlations found in Tress et al. (2018). We find that this simple model indeed produces an anticorrelation between $R_{V}$ and $B$, so that galaxies with a weaker NUV bump feature greyer attenuation, supporting earlier claims (see, e.g., Panuzzo et al. 2007, Noll et al. 2007. Buat et al. 2011, Narayanan et al. 2018).

Finally, we compared the observational data of SHARDS star-forming galaxies with the standard dust models of Draine \& Li (2007) and Witt \& Gordon (2000). Fig. 6 shows the best fits to the Draine \& Li (2007) models with respect to $E(B-V), R_{V}$ and $B$. The Draine \& Li (2007) parameters are the PAH fraction $\left(q_{\mathrm{PAH}}\right)$; the fraction of dust mass $(\gamma)$ that is exposed to starlight intensities $U_{\min }<U \leqslant U_{\max }$; the dust mass $\left(M_{d}\right)$; and the lower cutoff of the starlight intensity distribution $\left(U_{\min }\right)$. The increasing trend of $q_{\mathrm{PAH}}$ with $B$ and colour excess (Fig. 6) is suggestive of an increased contribution from $\mathrm{PAH}$ molecules in dustier environments with a more prominent bump strength Although weak, the data also reveal a correlation between dust mass and $E(B-V)$. Furthermore, we calculated the
$R_{V}$ and NUV bump strength equivalents of the Witt \& Gordon (2000) models. The results are shown in Table 1 and Fig. 7. As the optical depth increases, the attenuation becomes greyer and the bump strength weaker (Fig. 7, see also Salmon et al. 2016, Tress et al. 2018, Salim et al. 2018, Narayanan et al. 2018). We note that the observed anticorrelation between $R_{V}$ and $B$, i.e. greyer attenuation associated to a smaller bump, is also seen in other studies (Kriek \& Conroy 2013, Seon \& Draine 2016, Tress et al. 2018 Salim et al. 2018; Narayanan et al. 2018). Two alternative options can be considered to explain these correlations, invoking variations in either dust composition or dust geometry. Our analysis - based on simple extensions of the birth cloud model - suggests that geometry is potentially the major driver of these trends.

\section{REFERENCES}

Battisti A. J., Calzetti D., Chary R. R.,2016, ApJ, 818, 13 Battisti A. J., Calzetti D., Chary R. R., 2017, ApJ, 840, 109

Bruzual, G., Charlot., S., 2003, MNRAS, 344, 1000

Burgarella D., Buat V., Iglesias-Páramo J., 2005, MNRAS, 360, 1413

Buat, V., Giovannoli, E., Takeuchi, T. T., Heinis, S., Yuan, F.-T., Burgarella, D., Noll, S., Iglesias-Páramo, J., 2011, A\&A, 529, 22

Buat V., Noll S., Burgarella D., Giovannoli, E., et al., 2012, A\&A, 545, A141

Calzetti, D., Kinney, A.L., Storchi Bergmann, T. 1994 ApJ 429582

Calzetti, D., Armus L., Bohlin R. C.. 2000 ApJ 533682

Calzetti, D. 2001, PASP, 113, 1449

Cardelli J. A., Clayton G. C., Mathis J. S., 1989, ApJ, 345, 245

Cava, A., et al., 2015, ApJ, 812, 155

Charlot, S., Fall., S. M., 2000, ApJ, 539, 718 

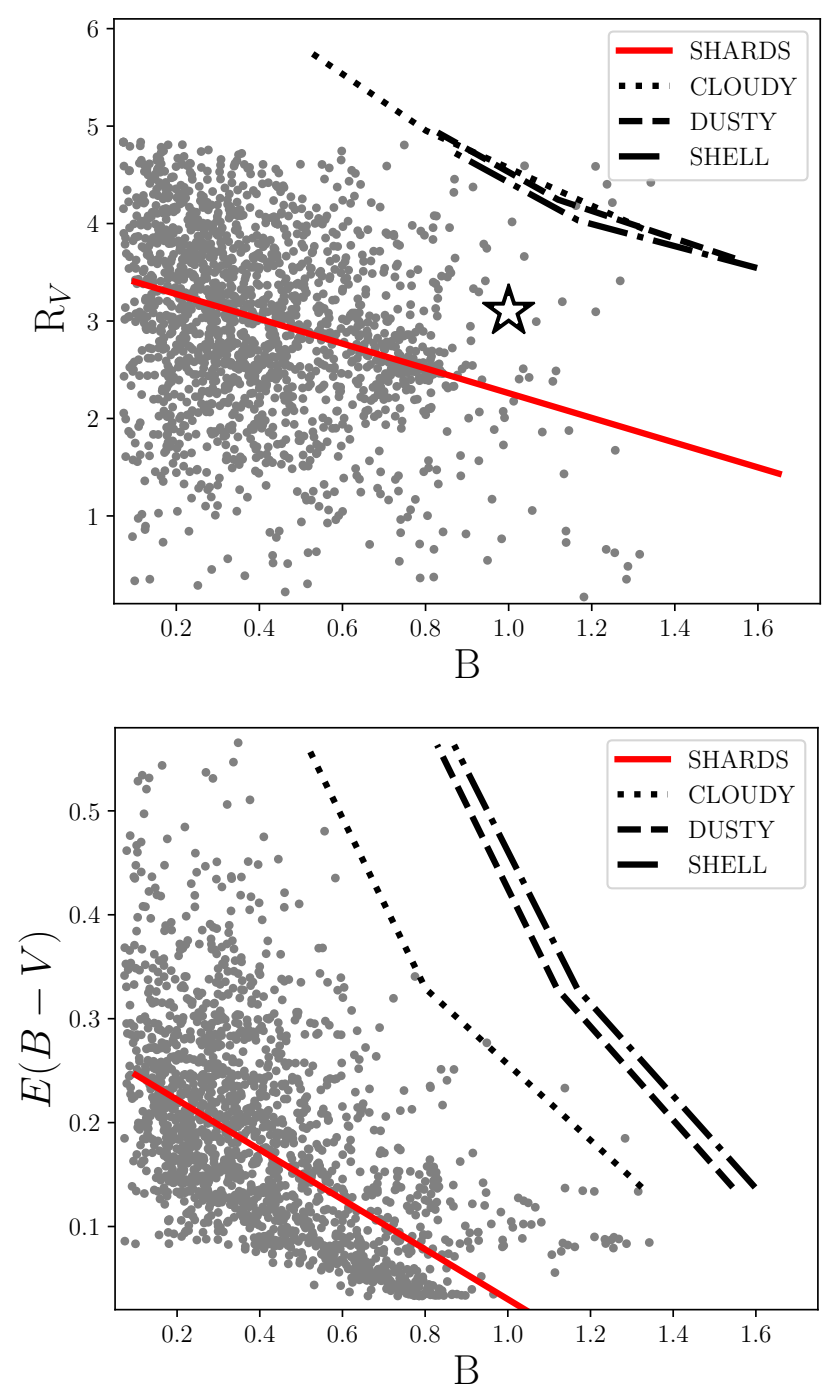

Figure 7. Comparison of the Witt \& Gordon (2000, WG00) models to the SHARDS star-forming galaxy sample of Tress et al. (2018) on the $R_{V}$ vs $B$ (top) and $E(B-V$ ) vs $B$ planes (bottom). Individual measurements are shown as grey dots, and the best fit to the data is given by the solid red line. The black lines, as labelled, correspond to the WG00 galactic environments, adopting a local clumpy structure in all three cases. For reference, the standard extinction law of the Milky Way is represented by a star in the top panel.

Clayton, G.C., Gordon, K.D., Bianchi, L.C., et al., 2015, ApJ, 815, 14

Conroy, C., Schiminovich, D., Blanton, M. R., 2010, ApJ, 718,184

Díaz-García L. A., et al., 2017, arXiv, arXiv:1711.10590

Domínguez Sánchez H., et al., 2016, MNRAS, 457, 3743

Draine B., 1989, IAUS, 135, 313

Draine B. T. \& Li, A., 2007, ApJ, 657, 810

Fasano G., Franceschini A., 1987, MNRAS, 225, 155

Fitzpatrick E.L., 1999, PASP, 111, 63

Fitzpatrick E. L., Massa D., 2007, ApJ, 663, 320

Foreman-Mackey D., Hogg D. W., Lang D., Goodman J., 2013, PASP, 125, 306

Galliano F., Galametz M., Jones A. P., 2018, ARA\&A, 56,
673

Gordon, K.D., Clayton, G.C., Misselt K.A., Landolt A.U., Wolff, M.J., 2003, ApJ, 594, 279

Groves B. A., Dopita M. A., Sutherland R. S., 2004, ApJS, 153,9

Hagen, L.M.Z., Siegel, M.H., Hoversten E.A., et. al., 2017, MNRAS, 466, 4540

Hutton, S. Ferreras, I., Yershov, V., 2015, MNRAS, 452, 1412

Johnson B. D., Schiminovich D., Seibert M. et al., 2007, ApJS, 173, 392

Kriek M., Conroy C. 2013 ApJL, 775, L16

Labbé I., et al., 2005, ApJ, 624, L81

Narayanan, D., Conroy, C., Davé, R., Johnson, B. \& Popping, G., 2018, ApJ, 869, 70

Natale, G., Popescu, C. C., Tuffs, R. J., Debattista, V. P., Fischera, J., Grootes, M. W., 2015, MNRAS, 449, 243

Noll S., Pierini D., Pannella M., Savaglio S., 2007, A\&A, 472, 455

Noll S., Burgarella D., Giovannoli E., Buat V., Marcillac D., Muñoz-Mateos J. C., 2009, A\&A, 507, 1793

Panuzzo, P., Granato, G. L., Buat, V.,et al., 2007, MNRAS, 375,640

Peacock, J. A., 1983, MNRAS, 202, 615

Pei Y. C., 1992, ApJ, 395, 130

Pérez-González P. G., Cava, A., Barro, G., et al., 2013, ApJ, 762, 46

Popescu C. C., Tuffs R. J., Dopita M. A., Fischera J., Kylafis N. D., Madore B. F., 2011, A\&A, 527, A109

Reddy N. A., Kriek M., Shapley A. E., et al., 2015, ApJ, 806, 259

Salim, S., Boquien,M. \& Lee, J., 2018, ApJ, 859, 11

Salmon, B., Papovich, C., Long, J., et al., 2016, ApJ, 827, 20

Seon, K.-I.,Draine, B. T., 2016, ApJ, 833, 201

Silva L., Granato G. L., Bressan A., Danese L., 1998, ApJ, 509, 103

Speagle, J. S., Steinhardt, C. L., Capak, P. L., Silverman, J. D., 2014, ApJS, 214, 15

Stecher T. P.,1969, ApJ, 157, L125

Tang J., Bressan A., Rosenfield P., Slemer A., Marigo P., Girardi L., Bianchi L., 2014, MNRAS, 445, 4287

Tang J., Bressan A., Slemer A., Marigo P., Girardi L., Bianchi L., Rosenfield P., Momany Y., 2016, MNRAS, 455,3393

Tress, M., Mármol-Queraltó, E., Ferreras, I., et al., 2018, MNRAS, 475, 2363

Whitaker K., Labbé, I., van Dokkum, P., et al., 2011, ApJ, 735,86

Wild V., Charlot S., Brinchmann J., et al., 2011, MNRAS, 417,1760

Williams R. J., Quadri R. F., Franx M., van Dokkum P., Labbé I., 2009, ApJ, 691, 1879

Witt, A. N., Gordon, K. D., 2000, ApJ, 528, 799

Wuyts S., et al., 2011, ApJ, 738, 106 